\title{
THE INFLUENCE OF PHYSIOTHERAPY ON FUNCTIONAL FITNESS OF ADULTS WITH DOWN SYNDROME
}

\author{
Ilona ILINCA ${ }^{1,2}$, Eugenia ROȘULESCU ${ }^{1,2}$, Germina COSMA ${ }^{1 *}$, Mircea DANOIU1 \\ ${ }^{1}$ University of Craiova, Faculty of Physical Education and Sport, Craiova, Romania \\ 2 "Sf. Maria" Neuromotor Adult Rehabilitation Centre, Craiova, Romania \\ *Corresponding author: germinacosma@yahoo.com
}

DOI: 10.35189/iphm.icpesk.2019.16

\begin{abstract}
Adults with Down syndrome possess a set of health, anatomical, physiological, cognitive and psycho-social attributes predisposing them to limitations on their physical fitness (aerobic capacity, muscular strength and endurance, flexibility and abnormal body composition), motor proficiency impairments (balance and postural control) and physical functional limitations. This study aimed to analyse the potential benefits of a physiotherapy intervention designed to improve certain aspects of functional fitness capacity among young adults with Down syndrome (DS) and mild-to-moderate mental retardation. Thirteen young adults with DS aged 19-25 were recruited from the "Sf. Maria" Neuromotor Adult Rehabilitation Centre in Craiova to perform the physiotherapy programme based on exercise therapy and aquatic therapy three or four times per week for six months. The measures of functional fitness capacity included: cardiopulmonary endurance (the six-minute walk test - 6MWT), muscular endurance (sit-up), flexibility (sit and reach), body composition (BMI, waist circumference), balance (Romberg test) and functional ability. Functional performance was assessed at baseline (week 0) and after 6 months following the therapy intervention. The results showed that, after completing the combined exercise programme, all the measures of functional fitness capacity were improved in post-tests compared to baseline assessments. The physiotherapy intervention appears to have a positive impact on the physical health of adults with DS, reducing the potential health risks associated with low fitness and a sedentary lifestyle, and therefore can be recommended as a non-pharmaceutical method.
\end{abstract}

Keywords: Down syndrome, physiotherapy intervention, benefits.

\section{Introduction}

In recent decades, the prognosis for people with Down syndrome has improved drastically. Many Down syndrome adults are healthier, more active in society, and have an increased life expectancy. This improved longevity has contributed to an increased focus on quality of life, especially as these individuals approach later stages of adulthood. However, average life expectancy is lower than in the rest of the population (O'Leary, Hughes-McCormack, Dunn, \& Cooper, 2018).

In addition to intellectual and developmental disabilities, there are some common health problems and some more serious medical conditions that are more likely to occur in people with Down syndrome than in other people. Adults with Down syndrome possess a set of health, anatomical, physiological, cognitive, and psycho-social attributes predisposing them to limitations on their physical fitness (aerobic capacity, muscular strength and endurance, flexibility and abnormal body composition), motor proficiency impairments (balance and postural control) and physical functional limitations.

The most common factors responsible for the low level of physical fitness of individuals with Down syndrome are a sedentary lifestyle (Merriman, Barnett, \& Jarry, 1996), low motivation for participation in physical activities (Halle, Gabler-Halle, \& Chung, 1999), or minimum opportunities, limited mental capacity and low attention levels.

Different studies have shown a general tendency towards decreased levels of physical activity in most teenagers and adults with Down syndrome (Rimmer, Heller, Wang, \& Valerio, 2004). This might be due to its association with overweight or obesity, osteoporosis, non-insulin dependent diabetes and cardiovascular disease (Prasher, 1995). Nearly 80 percent of those with Down syndrome are overweight, and individuals with DS are much more likely to die earlier from diabetes when compared to the general population (Rubin, Rimmer, Chicoine, Braddock, \& McGuire, 1998).

Promoting physical activity is a key component of controlling health status. It is widely accepted that physical activity can improve the physical fitness, work capacities and quality of life of individuals with intellectual disability. Additionally, regular physical activity of this people contributes to their mental and physical progress, increases motivation to live and emotional stability (Howie et al., 2012). For these reasons, young adults diagnosed with DS should be encouraged to participate in individual or group physical activity or exercise programmes. 
In view of these considerations, this study aimed to analyse the potential benefits of a physiotherapy intervention designed to improve certain aspects of functional fitness capacity among young adults with Down syndrome (DS) and mild-to-moderate mental retardation.

The hypothesis of this study is that an intervention using physiotherapy means (physical exercise) can improve certain aspects of functional fitness capacity and the anthropometric characteristics of patients with Down syndrome.

\section{Material and Methods}

The research used a one-group pretest-posttest design. The participants in the current study were recruited from the "Sf. Maria" Neuromotor Adult Rehabilitation Centre in Craiova, located in Southwest Romania. The "Sf. Maria" Centre is a modern rehabilitation clinic that offers rehabilitation services, being equipped with a medical office, 2 physical therapy treatment rooms, 2 electrical therapy rooms, a room for occupational therapy, a rehabilitation swimming pool and a psychological counselling room.

A total of 13 young adults diagnosed with Down syndrome (4 males and 9 females) aged 19 were recruited and allocated to the physiotherapy intervention group. Eligibility criteria included the following: (a) 18 years of age or older, (b) diagnosis of Down syndrome (c) written permission from primary care physician (d) IQ score between 45-70, (e) able to understand instructions and complete all tests, and (f) no difficulty with physical activity requiring musculoskeletal movement. Exclusion criteria included: contraindications to exercise; severe mental retardation; heart disease; metabolic disease; respiratory disorders; atlantoaxial instability and orthopaedic issues that would limit exercise performance. Subjects with open wounds or active infections, or who were fearful of being in a pool were also excluded. Before testing, all participants (or their parents) provided their written informed consent prior to their inclusion in the study, in accordance with the ethical principles of the Declaration of Helsinki.

After completing recruitment, pre-tests of functional fitness capacity of all participants were conducted prior to starting the intervention. The measures of functional fitness capacity included: cardiopulmonary endurance (the six-minute walk test - 6MWT), muscular endurance (sit-up), flexibility (sit and reach), body composition (BMI, waist circumference), balance (Romberg test) and functional ability (Gait speed test, Stair ascent and descent test). Functional performance was assessed at baseline - week 0 (pre) and after 6 months following the physiotherapy intervention (post). The results obtained are expressed as means and standard deviation (SD). The Paired t-Test was used in order to find the significance of the mean results.

All patients performed the physiotherapy programme based on exercise therapy and aquatic therapy three or four times per week for six months. During the exercise intervention, the subjects were instructed not to participate in any other form of exercise training. Local ethical permission was given by the Ethical Commission of the Faculty of Physical Education and Sport in Craiova.

\section{Intervention}

All subjects received a combined exercise programme including exercise therapy and aquatic therapy. Initially, they participated in the 40-minute aquatic exercise program, and subsequently, in the 45-minute land-based exercise program. There was a 30-minute rest and a meal (sandwich) break between the two different therapies.

The aquatic exercise intervention developed for the current research was designed following the review of the recent literature. In order to comply with the physical abilities, needs and desires of participants, we consulted two specialists in the field of adapted physical activities. The aquatic exercise was performed in the swimming pool of the "Sf. Maria" Neuromotor Adult Rehabilitation Centre. The size of the pool is $12 \mathrm{~m}$ by $5 \mathrm{~m}, 1.4 \mathrm{~m}$ depth, heated to $32-32.5^{\circ} \mathrm{C}$, respecting the hygiene rules. The aquatic exercise programme was carried out three or four times per week for 6 months, with at least 2 days between sessions, and each aquatic exercise session lasted 45-50 minutes. A qualified physical therapist supervised all pool sessions. In addition, 3-5 volunteer students assisted patients with the exercises and ensured safety and optimal participation.

Exercises were performed with gradual increases in intensity and duration according to each patient's ability. The intensity of aquatic aerobic exercise was set according to the recommendations of the American College of Sports Medicine (Schmitz et al., 2010) and through the use of the Borg Rating of Perceived Exertion Scale (RPE). Endurance exercises were performed at a moderate intensity, 2-3 sets for each exercise of 8-12 repetitions per set. 
They followed a normal sequence of warm-up, conditioning, strengthening, and cool-down routines. The general aims of the combined exercise programme were to increase functional fitness capacity and maintain optimal health. Individual aims consisted of improving cardiopulmonary function, muscle strength and endurance, flexibility of the muscle-joint structures, coordination and balance, body composition and functional activities.

A 5-minute warm-up was performed in the pool and included walking in the water in various directions and stretching exercises. The main part included cardiovascular exercises initially based on walking in shallow (waistdeep) water and then increasing water depth and speed, forward and backward jogging with different movements of the arms and hopping movements in multiple directions. The joint mobility and muscular strength exercises focused on movements for the primary joints and movements for the lower/upper body and trunk strengthening using different resistive devices. Aquatic strengthening exercises were progressed by adding more resistance or weight, increasing the number of repetitions or sets, varying the support surface during balance exercises and increasing the duration of the aquatic programme. To enhance balance and proprioception, we included in the aquatic programme unilateral and bilateral exercises, stepping in different directions, turning tasks (180-degree turn) and movements on the Kickboard and Ankle Board. We established a couple of different variables to diversify the balance challenge: reducing the size of the base of support, the amount of assistance required, external supports and head position. Finally, at the end of each session, the subjects did stretching/flexibility activities and breathing exercises for 5 minutes to return to their initial state. The exercises for each section are summarised in Table 1.

The land-based exercise programme followed the same sequences and objectives as the aquatic intervention. The exercise training focuses on joints in the trunk, shoulders, arms and legs, and emphasises the muscle groups of the upper and lower limbs, as well as balance and coordination. Movement against gravity, hand and ankle weights, gradual increases in the average number of repetitions for each exercise and variations in speed were used to create different levels of programme intensity. The exercises for each section are summarised in Table 1.

Table 1. Aquatic and land-based exercise programmes

\begin{tabular}{ll|ll}
\hline & \multicolumn{1}{c|}{$\begin{array}{c}\text { Aquatic programme } \\
\text { Type of exercises }\end{array}$} & & $\begin{array}{c}\text { Land-based programme } \\
\text { Type of exercises }\end{array}$ \\
\hline Warm-up & $\begin{array}{l}\text { Forward walking, backward walking, } \\
\text { sidestepping, lunging, toe and heel } \\
\text { walking, marching; }\end{array}$ & $\begin{array}{l}\text { Warm-up } \\
(5-7 \text { min })\end{array}$ & $\begin{array}{l}\text { Gentle muscle stretches, gentle } \\
\text { endurance exercises including cycle } \\
\text { ergometer for the lower limbs. }\end{array}$ \\
& Stretching exercises; & &
\end{tabular}

Cardiovascular Forward walking with shoulders in exercises horizontal abduction, then with kickboard in front, then with trunk rotation; tandem walking; walking sideways with long steps; Forward and backward jogging with larger arm movements or crossing the midline of the body, and combined hopping in opposite directions; Performed with or without resistance paddles -2 sets of 10 repetitions each.

Joint mobility Shoulder flexion-extension, abductionand muscular adduction; Biceps/triceps exercises; strength exercises Wall Push-ups;

Kickboard exercises - Press-downs; Chest press;

Trunk rotation, trunk side-bending; Hip movements: flexion, extension, abduction, adduction;
Marching and sidestepping with variations in the directions of movement, arm movements; Elliptical; Walking on the treadmill tilted to $4 \%$ or $8 \%$ and $12 \%$;

Step-ups, Step-downs;

3 minutes of continuous activity; (110-125 beats/min) with 2 min rest between aerobic exercises.

Repeat the exercises as in the aquatic section.

Exercises were performed in a standing position using a stall bar for support and resistance tubing to strengthening.

Each exercise lasts 35-40 s; 2 sets of each exercise for 10 to 15 repetitions. 


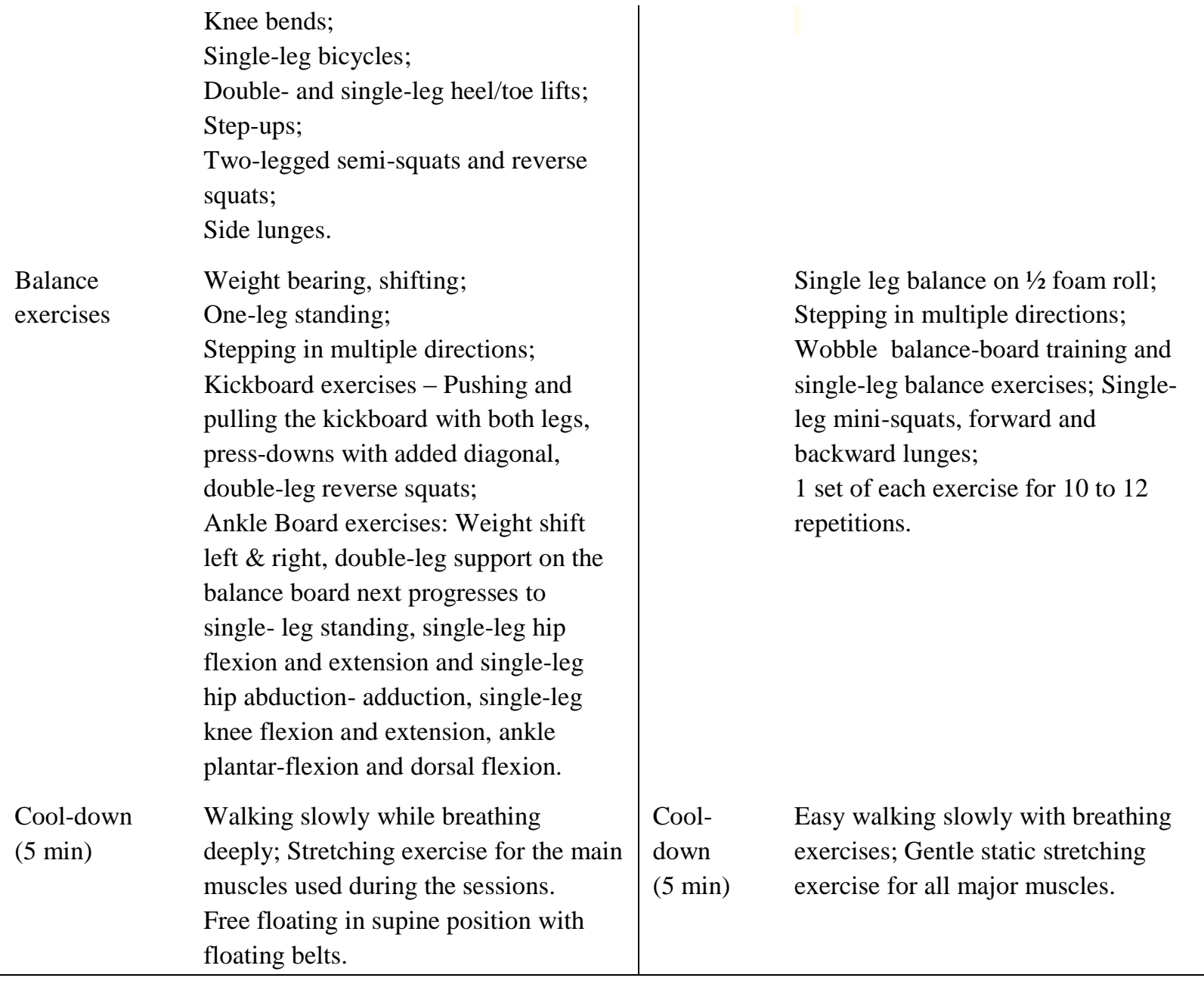

Cardiopulmonary endurance and functional exercise capacity were assessed using the 6-minute walk test (6MWT). In recent years, the 6MWT has been tested with clinical populations, as well as adults with mental retardation, offering a satisfactory insight into the functional status of participants' bodies, which is necessary for daily physical activities (Enright, 2002). The test was performed along a straight, long, flat, enclosed 30-m corridor with a hard surface. The participants were instructed to walk as fast as possible to cover the longest distance possible within 6 minutes at their own pace, but without running. The distance covered within the 6 min was measured to the nearest meter.

Abdominal muscle strength was assessed by the sit-up test. The patients were asked to lie supine with $90^{\circ}$ flexion in the knee joints and hands placed on the side of their head. For 30 seconds, they continually sat up to touch their knees with the elbows. The test counts the number of correct sit-ups completed within 30 seconds.

Flexibility of the lower back and hamstring muscles was measured using the sit-and-reach test. The subjects were asked to assume long sitting position with legs stretched out straight ahead with the bottom of their bare feet against the end of the box. Then, the participants were instructed to extend their arms and put one hand over the other on the top of the box where a measuring tape was placed, and after that they were instructed to reach forward as far as possible on the measuring tape while maintaining their knees, arms and fingers fully extended and one hand over the other. The score was recorded as the most distant point on the measuring tape contacted by the finger.

Anthropometric measurement, such as patient's weight and height, was also performed. The body mass index (BMI) [weight $(\mathrm{kg}) /$ height $\left(\mathrm{m}^{2}\right)$ ] was calculated. Waist circumference was measured with the subjects in a standing position using a flexible tape taken midway between the 10th rib and the iliac crest.

The ability to maintain static balance under conditions of reduced base of support was measured using the Romberg test (Yim-Chiplis \& Talbot, 2002). In the test, the subjects remained standing up with the feet together in parallel position, the arms to the side and the eyes closed for 60 seconds. A loss of balance and the inability to maintain the initial position of the test indicated a change in static balance for the Romberg test. 
Each patient was timed on their ability to perform two functional tasks of daily living as fast as comfortably possible, which included gait speed and the time to ascend and descend 10 steps.

Gait speed (GS) is a valid and reliable measure appropriate for assessing functional status and overall health in a wide range of populations (Bohannon, 1997). Gait speed measures the time it takes the participant to walk a predetermined short distance $(7.62 \mathrm{~m})$. The stair ascent and descent test assesses the subjects' ability to ascend 10 stairs as quickly as possible, and then to descend the 10 steps as fast as possible. The time taken to complete the ascent and descent tests was recorded.

\section{Results}

The study used a one-group pretest-posttest design. Descriptive statistics were calculated, and the Paired t-Test was performed in order to find the significance of the mean results. To this purpose, we used the SPSS program, version 21.

Table 2 shows the means and standard deviation (SD) for pre- and post-exercise results. They reveal that the training group has improved their results in all physical fitness measures (except height), the greatest gains being in body strength and flexibility.

Table 2. Descriptive statistics for pre- and post-exercise results

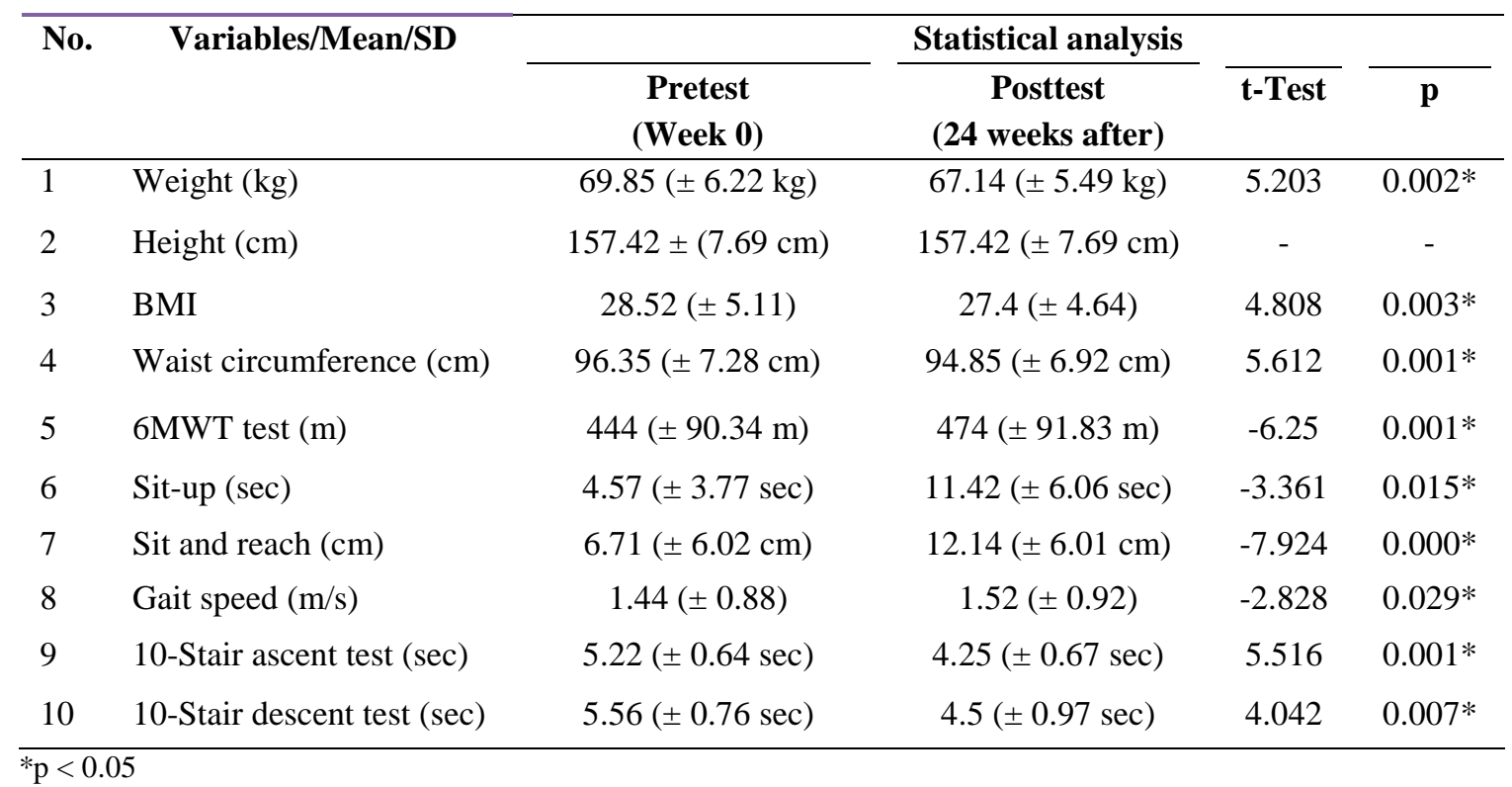

Before exercise, $80 \%$ of participants were overweight (BMI over 25) and $20 \%$ were obese (BMI over 30). In the pretest results, the subjects had a BMI mean of $28.52( \pm 5.11)$, and posttest, $27.4( \pm 4.64)$. After the combined exercise programme, the improvement is significant $(\mathrm{t}-4.808, \mathrm{p}<0.05)$. Waist circumference decreased by almost $2 \mathrm{~cm}$ in posttest $(\mathrm{t}-4.808, \mathrm{p}<0.05)$. Regarding the six-minute walk test $(6 \mathrm{MWT})$, significant differences are noted between pre- and posttest $(\mathrm{t}-6.25, \mathrm{p}<0.05)$, all subjects improving their cardiopulmonary endurance.

The sit-up test recorded the value $4.57( \pm 3.77 \mathrm{sec})$ at the beginning of the exercise programme, and $11.42( \pm$ $6.06 \mathrm{sec})$ at its end. Mean differences were significant at $\mathrm{p}=0.015$. Significant mean differences were noted in the sit-and-reach test $(\mathrm{t}-7.924, \mathrm{p}<0.001)$, all subjects showing substantial gains in muscle flexibility.

Compared to posttest results, a significant difference was observed for gait speed $(\mathrm{t}-2.82, \mathrm{p}<0.05)$.

The functional ability to ascent and descent the stairs showed significant improvements $(\mathrm{p}<0.05)$ after 24 weeks of physical exercise.

All participants were assessed at baseline using the Romberg test. The test was negative for all patients, who managed to maintain their static balance for more than 60 seconds.

\section{Discussion}


Our sample of adults with Down syndrome improved their results in all measures of functional fitness capacity, with statistically significant gains noted for the 6MWT, sit-up and sit-and-reach tests. Lower cardiovascular levels reported in adults with Down syndrome may be associated with greater susceptibility to functional decline, indicating that they may be predisposed to a higher incidence of cardiovascular disease and other health complications. Cardiovascular conditioning and strength are extremely important fitness components for both maintaining optimal cardiovascular health and proper musculoskeletal function to conduct various tasks with less physiological stress and preventing participation restrictions specifically related to job performance.

The 6-month programme of combined exercises based on exercise therapy and aquatic therapy resulted in an improvement of the ability to cover a longer distance during the 6MWT. Relying on the significant differences found, we can consider that the structure, period and intensity of the physiotherapy intervention programme have induced an improvement in the level of cardiopulmonary endurance among the participants with DS.

There is some evidence in the literature that shows changes in the cardiopulmonary endurance in adults with intellectual disability after an exercise programme. Tsimaras, Giagazoglou, Fotiadou, Christoulas and Angelopoulou (2003) assessed the effects of an aerobic training programme on 25 adults with Down syndrome. The authors concluded that adults with Down syndrome demonstrated a significantly greater increase $(\mathrm{p}<.05)$ in all physiological parameters compared with the non-exercise control group. Varela, Sardinha and Pitetti (2001) investigated the effects of a rowing exercise regimen on cardiovascular fitness of young adults with Down syndrome. They found that the intervention group was capable of walking and rowing longer distances $(\mathrm{p}<.05)$ following their involvement in the aerobic programme, demonstrating increases in exercise capacity without improvements in aerobic fitness.

Muscular endurance is essential for daily living and recreational activities, as well as for work capacity. Muscle flexibility is negatively influenced by the sedentary lifestyle, gender, age, muscle imbalances, but also the deterioration of joint and cartilage structures. Muscle flexibility is necessary for the unrestricted performance of daily living activities in the DS population. In the present study, abdominal muscle endurance was assessed by the sit-up test, and hamstring flexibility was assessed by the sit-and-reach test. An improved trend in some physical fitness results (e.g., sit and reach and sit-up) was observed, with statistically significant differences after the combined physical and aquatic therapy. Similar results were also obtained by $\mathrm{Wu}$ et al. (2010), who found decreases in the subjects' weight and BMI scores and positive values in the sit-and-reach and 30- and 60-sec sit-up tests following the long-term exercise programme for adults with intellectual disability.

Balance is one of the abilities in which individuals with DS are most deficient. Although not a goal for healthrelated fitness balance, it is important in many physical activities like walking and climbing stairs and also has health implications. In this study, the ability to maintain static balance was measured using the Romberg test. The assessed subjects had the ability to maintain their initial position for more than 60 seconds.

Walking fast and going up and down the stairs are important functional measures of ability and are necessary for independent living. In our study, the ability to increase the walking speed and the performance of ascending and descending stairs may be related to improved functional ability following completion of the 6-month programme of combined exercises.

\section{Conclusion}

The results showed that, after completing the combined exercise programme, all the measures of functional fitness capacity were improved in post-tests compared to baseline assessments. The duration of an exercise programme is undoubtedly an essential aspect and has significant importance for the results obtained. The physiotherapy intervention appears to have a positive impact on the physical health of adults with DS, reducing the potential health risks associated with low fitness and a sedentary lifestyle and improving the ability to perform tasks relevant to everyday life, and therefore can be recommended as a non-pharmaceutical method.

Further studies should determine the effects of reduced functional fitness level on the ability of people with Down syndrome to perform everyday activities, because such research can be helpful in designing effective physiotherapy interventions.

\section{References}


Bohannon, R. W. (1997). Comfortable and maximum walking speed of adults aged 20-79 years: Reference values and determinants. Age Ageing, 26(1), 15-19. https://doi.org/10.1093/ageing/26.1.15

Enright, P. L. (2002). The six-minute walk test. Respiratory Care, 48(8), 783-785. Retrieved from https://www.ncbi.nlm.nih.gov/pubmed/12890299

Halle, J. W., Gabler-Halle, D., \& Chung, Y. B. (1999). Effects of a peer-mediated aerobic conditioning program on fitness levels of youth with mental retardation: Two systematic replications. Mental Retardation, 37(6), 435-448. https://doi.org/10.1352/0047-6765(1999)037\%3C0435:EOAPAC\%3E2.0.CO;2

Howie, E. K., Barnes, T. L., McDermott, S., Mann, J. R., Clarkson, J., \& Meriwether, R. A. (2012). Availability of physical activity resources in the environment for adults with intellectual disabilities. Disability and Health Journal, 5(1), 41-48. https://doi.org/10.1016/j.dhjo.2011.09.004

Merriman, W. J., Barnett, B. E., \& Jarry, E. S. (1996). Improving fitness of dually diagnosed adults. Perceptual and Motor Skills, 83(3 Pt. 1), 999-1004. https://doi.org/10.2466/pms.1996.83.3.999

O’Leary, L., Hughes-McCormack, L., Dunn, K., \& Cooper, S. A. (2018). Early death and causes of death of people with Down syndrome: A systematic review. Journal of Applied Research in Intellectual Disabilities, 31(5), 687-708. https://doi.org/10.1111/jar.12446

Prasher, V. P. (1995). Overweight and obesity amongst Down's syndrome adults. Journal of Intellectual Disability Research, 39(5), 437-441. https://doi.org/10.1111/j.1365-2788.1995.tb00548.x

Rimmer, J. H., Heller, T., Wang, E., \& Valerio, I. (2004). Improvements in physical fitness in adults with Down syndrome. American Journal on Mental Retardation, 109(2), 165-174. https://doi.org/10.1352/08958017(2004)109\%3C165:IIPFIA\%3E2.0.CO;2

Rubin, S. S., Rimmer, J. H., Chicoine, B., Braddock, D., \& McGuire. D. E. (1998). Overweight prevalence in persons with Down syndrome. Mental Retardation, 36(3), 175-181. https://doi.org/10.1352/00476765(1998)036\%3C0175:OPIPWD\%3E2.0.CO;2

Schmitz, K. H., Courneya, K. S., Matthews, C., Demark-Wahnefried, W., Galvão, D. A.,.. \& Schwartz, A. L. (2010). American College of Sports Medicine roundtable on exercise guidelines for cancer survivors. Medicine \& Science in Sports \& Exercise, 42(7), 1409-1426. https://doi.org/10.1249/MSS.0b013e3181e0c112

Tsimaras, V., Giagazoglou, P., Fotiadou, E., Christoulas, K., Angelopoulou, N. (2003). Jog-walk training in cardiorespiratory fitness of adults with Down syndrome. Perceptual and Motor Skills, 96(3 Pt. 2), 1239-1251. https://doi.org/10.2466/pms.2003.96.3c.1239

Varela, A. M., Sardinha, L. B., \& Pitetti, K. H. (2001). Effects of an aerobic rowing training regimen in young adults with Down syndrome. American Journal on Mental Retardation, 106(2), 135-144. https://doi.org/10.1352/0895-8017(2001)106\%3C0135:EOAART\%3E2.0.CO;2

Wu, C. L., Lin, J. D., Hu, J., Yen, C. F., Yen, C. T., Chou, Y. L., \& Wu, P. H. (2010). The effectiveness of healthy physical fitness programs on people with intellectual disabilities living in a disability institution: Six-month short-term effect. Research in Developmental Disabilities, $713-717$. https://doi.org/10.1016/j.ridd.2010.01.013

Yim-Chiplis, P. K., \& Talbot, L. A. (2000). Defining and measuring balance in adults. Biological Research for Nursing, 1(4), 321-331. https://doi.org/10.1177/109980040000100408 